\title{
Checklist of oribatid mites (Acari: Oribatida) in the Wigry National Park, North-East Poland
}

\author{
Josef STARÝ \\ Institute of Soil Biology, Biology Centre v.v.i., Czech Academy of Sciences, Na Sádkách 7, CZ - 37005, České \\ Budějovice, Czech Republic; e-mail: jstary@upb.cas.cz
}

\begin{abstract}
Detailed faunistic research of soil oribatid mites was caried out at 11 localities representing important forest types in the territory of Wigry National Park. Altogether 140 oribatid species were found in this territory, belonging to 42 families and 80 genera or subgenera; from them 127 oribatid species were found on the territory NP Wigry for the first time. Records of four species Brachychthonius impressus Moritz, 1976, Quadroppia monstruosa Hammer, 1979, Suctobelba regia Moritz, 1970 and Zetorchestes flabrarius Grandjean, 1951 are new for the fauna of Poland.
\end{abstract}

Key words: soil mites, new records, fauna, forest habitats, distribution list

\section{INTRODUCTION}

Oribatid mites (Acari: Oribatida) is a suborder of mites with the highest species diversity and abundance in soil and above all in the upper litter layers (Norton 1985, Behan-Pelletier \& Newton 1999). More than 10000 species have been described worldwide so far (Schatz 2002) and their abundance in forest soils reaches tens to several hundred thousand individuals per $\mathrm{m}^{2}$ (Petersen \& Luxton 1982).

This ubiquitous group of soil invertebretes is sensitive to environmental changes and their activity significantly affects the decomposition of organic matter in the soil (Abbott \& Crossley 1982, Seastedt 1984, Heneghan et al. 1999), mineralization (Beare et al. 1992), nutrient cycle in the soil (Seastedt \& Crossley 1988, Setälä \& Huhta 1991) and the development of soil texture and microstructure (Maraun et al. 1998). They are therefore considered to be important bioindicators of functional relationships in the soil (Loreau et al. 2001, Paoletti et al. 2007)

The study of oribatid mites has a long tradition in Poland and oribatid fauna is relatively well known, especially in some areas of this country. Oribatida of the Wigry National Park have so far been studied only marginally and nolist of species inhabiting major NP Wigry biotops has been published yet. Olszanowski et al. (1996) and Niedbała \& Olszanowski (2008) published lists of all oribatid species found in Poland. In total, there are findings of 505 species belonging to 78 families and 171 genera and subgenera. Two brachychthonid species were recorded previously (Niedbała 1976) from Wigry NP and Niedbała (2008) presented records of 10 ptyctimous oribatid species from the pine forest between Krzywe and Stary Folwark and from the Poddubówek locality. Olszanowski et al. (1996) presented record of eurytopic species Tectocepheus velatus from locality Poddubówek. In total, the following 13 species of oribatid mites has been known from the NP Wigry so far: Acrotritia ardua (C. L. Koch, 1841), Atropacarus csiszarae (Balogh \& Mahunka, 1979), Euphthiracarus cribrarius (Berlese, 1904), Phthiracarus boresetosus Jacot, 1930, P. bryobius Jacot, 1930, P.ferrugineus (C. L. Koch, 1841), P.longulus (C. L. Koch, 1841), P.nitens (Nicolet, 1855), Sellnickochthonius jacoti (Evans, 1952), Synchthonius crenulatus (Jacot, 1938), Steganacarus applicatus (Sellnick, 1920), S. carinatus (C. L. Koch, 1841), and Tectocepheus velatus (Michael, 1880). 


\section{MATERIAL AND METHODS}

Altogether 280 quantitative soil samples were collected during 2016-2019 on 11 localities within the Wigry National Park. Quantitative soil samples were collected using cylindrical steel corer with workspace $10 \mathrm{~cm}^{2}, 10 \mathrm{~cm}$ in depth. Always five separate soil samples were collected from each studied plot. The corer has outer steel case with inner plastic longitudinally divided case allowing the obtaining of intact soil monolith. Collected soil samples were enclosed in plastic bags to prevent them from desication and quickly transferred to the laboratory. Soil mites from collected soil samples were extracted using a high efficiency „high gradient" fototermoextractor, modification along Marshall (1972).

\section{Study area and localities}

The Wigry National Park designated in 1989, covers an area of $150.8 \mathrm{~km}^{2}$ with a further $118.2 \mathrm{~km}^{2}$ forming its buffer zone, is located in the north-western part of the Augustów Forest in the Podlaskie voivodeship. The park is largely a forested area $\left(94.6 \mathrm{~km}^{2}, 62.7 \%\right)$. Scots pine (Pinus sylvestris) stands prevail in the forests, whilst in the smaller forest expanses, there dominates Norway spruce (Picea abies), black alder (Alnus glutinosa), downy birch (Betula pubescens) or pedunculate oak (Quercus robur). The remaining portion of the park consists of waters $\left(29.1 \mathrm{~km}^{2}, 19.3 \%\right)$ and non-forest communities $\left(27.1 \mathrm{~km}^{2}, 18.0 \%\right)$. The area remains under the influence of continental climate characterised by long winters. The annual average temperature is $6.3{ }^{\circ} \mathrm{C}$; the annual average precipitation is $589 \mathrm{~mm}$; NP lies at an altitude 132178 m (Loziński 2002).

Soil samples were collected from area near Sobolewo and Krzywe at the following 11 sites:

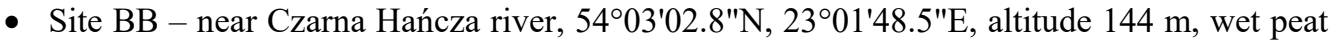
forest with dominant Scots pine (Pinus sylvestris), with Sphagnum spp., Ledum palustre, Vaccinium uliginosum and Eriophorum vaginatum.

- Site BE - forest along Czarna Hańcza river, 5402'45.8"N, 2302'47.9"E, altitude $143 \mathrm{~m}$, wet peat forest with dominant downy birch (Betula pubescens) and Scots pine (Pinus sylvestris).

- Site GR - along the Czarna Hańcza river, 5403'32.0"N, 2301'04.5"E, altitude $167 \mathrm{~m}$, deciduous and partly mixed forest (Tilio-Carpinetum) on higher terrace with common hazel (Corylus avellana) and Norway spruce (Picea abies).

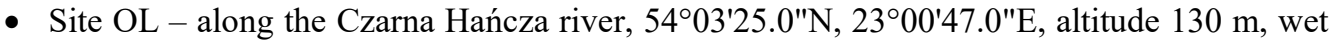
alder forest (Alnetum) with dominant black alder (Alnus glutinosa).

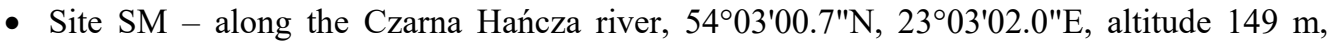
waterlogged peat spruce forest (Picetum) with dominating Sphagnum spp. and bilberry (Vaccinium myrtillus).

- Site Imp 1 - deciduous forest, $54^{\circ} 01^{\prime} 56.8^{\prime}$ N, longitude $22^{\circ} 59$ '34.2”E, altitude $167 \mathrm{~m}$, plant community - Tilio-Carpinetum calamagrostietosum with Plagiomnium affine, site with the highest density of Impatiens parviflora.

- Site Imp5 - deciduous forest, 5404'22.7” N, 2300'55.0”'E, altitude 149 m, plant community - Tilio-Carpinetum typicum, site with a high density of Impatiens parviflora.

- Site Imp6 - deciduous forest, 5404'29.5” N, 2300'59.4”E, altitude $161 \mathrm{~m}$, plant community - Tilio-Carpinetum typicum, site with low density of Impatiens parviflora.

- Site Imp4a - deciduous forest, 5401'51.1” N, 2301'27.4”E, altitude $154 \mathrm{~m}$, plant community - Tilio-Carpinetum calamagrostietosum typicum, control plot without Impatiens parviflora.

- Site Imp4b - deciduous forest, 5401'57.0” N, 2300’56.0”E, altitude $164 \mathrm{~m}$, plant community - Tilio-Carpinetum typicum, control plot without Impatiens parviflora. 
- Site Imp $4 \mathrm{c}$ - decidous forest, $54^{\circ} 01^{\prime} 54.6^{\prime \prime} \mathrm{N}, 23^{\circ} 01^{\prime} 06.3^{\prime \prime} \mathrm{E}$, altitude $161 \mathrm{~m}$, plant community

- Tilio-Carpinetum calamagrostietosum typicum, control plot without Impatiens parviflora.

Oribatid mites were cleared before determination in $80 \%$ solution of lactic acid. The species determination was carried out under the Leica DMR microscope individually on the temporary slides with small hollow, as it was necessary to observe the individuals from different sides and angles. Determined oribatid species were preserved in glycerol in Terasaki microtitration boxes and deposited in collection of ISB in České Budějovice. The following keys were used for oribatid mite determination: (Sellnick 1928, 1960, Willmann 1931, Kunst 1971, Giljarov et al. 1975, Balogh \& Mahunka 1983, Weigmann 2006, Niedbała 2011). The checklist follows classification used by Weigmann (2006). Distribution of recorded species follows mainly data published by Subias (2004).

\section{TAXONOMIC CHECKLIST OF ORIBATID MITE SPECIES FOUND IN THE WIGRY NATIONAL PARK}

Altogether rich material of 25673 individuals of oribatid mites was obtained. In total 140 oribatid species were found in Wigry NP, belonging to 42 families and 80 genera or subgenera. Records of six species: Acrotritia ardua, Euphthiracarus cribrarius, Sellnickochthonius jacoti, Steganacarus applicatus, S. carinatus, and Tectocepheus velatus, known earlier from NP Wigry were confirmed, other 127 oribatid species were found on the territory NP Wigry for the first time and finaly, records of four oribatid mites, Brachychthonius impressus, Quadroppia monstruosa, Suctobelba regia and Zetorchestes flabrarius are new for the fauna of Poland.

Palaeacaridae Grandjean, 1932

1. Palaeacarus hystricinus Trägårdh, 1932

Distribution: Holarctis, South-East China.

Locality in NP Wigry: Imp5.

Ctenacaridae Granjean, 1954

\section{Aphelacarus acarinus (Berlese, 1910)}

Distribution: semicosmopolitan (Holarctis, Etiopian and Neotropical Regions).

Localities in NP Wigry: Imp1.

Brachychthoniidae Thor, 1934

\section{Brachychthonius berlesei Willmann, 1928}

Distribution: Holarctis.

Locality in NP Wigry: Imp4c.

\section{Brachychthonius bimaculatus Willmann, 1936}

Distribution: Holarctis.

Localities in NP Wigry: Imp4c, Imp6.

\section{Brachychthonius hirtus Moritz, 1976}

Distribution: Central and South Europe, Iran and Mongolia.

Locality in NP Wigry: Imp4c. 


\section{Brachychthonius impressus Moritz, 1976}

Distribution: Palaearctis.

Localities in NP Wigry: GR, Imp1, Imp4b, Imp4c.

New record for Poland.

\section{Brachychthonius pius Moritz, 1976}

Distribution: Holarctis.

Locality in NP Wigry: Imp4a.

\section{Eobrachychthonius oudemansi van der Hammen, 1952}

Distribution: semicosmopolitan.

Locality in NP Wigry: Imp4b.

\section{Liochthonius brevis (Michael, 1888)}

Distribution: Holarctis.

Localities in NP Wigry: BE, SM, Imp4a, Imp4c, Imp5.

\section{Liochthonius horridus (Sellnick, 1928)}

Distribution: Palaearctis.

Locality in NP Wigry: Imp1.

\section{Liochthonius hystricinus (Forsslund, 1942)}

Distribution: Holarctis and Chile.

Localities in NP Wigry: Imp1, Imp4a.

\section{Liochthonius sellnicki (Thor, 1930)}

Distribution: Holarctis and South-East China.

Localities in NP Wigry: GR, Imp1, Imp4b, Imp4c, Imp5, Imp6.

\section{Liochthonius strenzkei Forsslund, 1963}

Distribution: Holarctis.

Locality in NP Wigry: Imp4c.

\section{Liochthonius tuxeni (Forsslund, 1957)}

Distribution: Europe and Iran.

Locality in Wigry NP: GR.

\section{Mixochthonius pilososetosus (Forsslund, 1942)}

Distribution: Palaearctis and Canada.

Locality in NP Wigry: SM.

\section{Sellnickochthonius immaculatus (Forsslund, 1942)}

Distribution: Holarctic and Neotropical Region.

Localities in NP Wigry: GR, Imp1, Imp4a, Imp4b, Imp4c, Imp5, Imp6.

\section{Sellnickochthonius jacoti (Evans, 1952)}

Distribution: Palaearctis and USA.

Localities in NP Wigry: Folwark (Niedbała 1976, Olszanowski et al. 1996), Imp1, Imp4b, Imp4c, Imp5. 


\section{Sellnickochthonius rostratus (Jacot, 1936)}

Distribution: Holarctis.

Localities in NP Wigry: BB, GR, Imp1, Imp4a, Imp4b, Imp4c, Imp5, Imp6.

\section{Sellnickochthonius suecicus (Forsslund, 1942)}

Distribution: semicosmopolitan (Palaearctis, Nord Nearctis, Australia, New Zealand).

Localities in NP Wigry: GR, Imp1, Imp4b, Imp4c, Imp5.

\section{Sellnickochthonius zelawaiensis (Sellnick, 1928)}

Distribution: Holarctis.

Localities in NP Wigry: BB, Imp1, Imp5.

\section{Synchthonius crenulatus (Jacot, 1938)}

Distribution: Holarctis.

Localities in NP Wigry: Poddubówek (Niedbała 1976, Olszanowski et al. 1996).

Hypochthoniidae Berlese, 1910

\section{Hypochthonius rufulus C. L. Koch, 1835}

Distribution: semicosmopolitan (Holarctis, Oriental Region, Seychelles, Mexico).

Localities in NP Wigry: OL, BE, BB, GR, SM, Imp4c.

Eniochthoniidae Grandjean, 1947

\section{Eniochthonius minutissimus (Berlese, 1903)}

Distribution: cosmopolitan.

Localities in NP Wigry: OL, GR, Imp1, Imp4a, Imp4b, Imp4c, Imp5, Imp6.

Eulohmanniidae Grandjean, 1931

24. Eulohmannia ribagai (Berlese, 1910)

Distribution: Holarctis and Oriental Region.

Locality in Wigry NP: SM.

Phthiracaridae Perty, 1841

25. Atropacarus (Atropacarus) csiszarae (Balogh et Mahunka, 1979)

Distribution: Central and South Europe.

Localities in NP Wigry: Stary Folwark and Krzywe (Olszanowski et al. 1996, Niedbała 2008).

26. Atropacarus (Atropacarus) striculus (C. L. Koch, 1835)

Distribution: semicosmopolitan (Holarctic, Oriental Region, Central America, Australia). Localities in NP Wigry: OL, BE, BB, GR, SM, Imp1, Imp4a, Imp4b, Imp4c, Imp5, Imp6.

\section{Phthiracarus boresetosus Jacot, 1930}

Distribution: semicosmopolitan.

Localities in NP Wigry: Stary Folwark and Krzywe, (Olszanowski et al. 1996, Niedbała 2008). 


\section{Phthiracarus bryobius Jacot, 1930}

Distribution: Holarctis, St. Helena Island, Nepal, Costa Rica and Bolivia.

Locality in NP Wigry: Poddubówek, (Olszanowski et al. 1996, Niedbała 2008).

\section{Phthiracarus ferrugineus (C. L. Koch, 1841)}

Distribution: West Palaearctis and Sri Lanka.

Locality in NP Wigry: Poddubówek, (Olszanowski et al. 1996, Niedbała 2008)

30. Phthiracarus longulus (C. L. Koch, 1841)

Distribution: Holarctis and Uruguay.

Localities in NP Wigry: Stary Folwark, Krzywe and Poddubówek, (Olszanowski et al. 1996, Niedbała 2008)

\section{Phthiracarus nitens (Nicolet, 1855)}

Distribution: Palaearctis.

Localities in NP Wigry: Stary Folwark and Krzywe (Olszanowski et al. 1996, Niedbała 2008).

32. Steganacarus (Steganacarus) applicatus (Sellnick, 1920)

Distribution: West of Palaearctis.

Localities in NP Wigry: Stary Folwark, Krzywe and Poddubówek (Olszanowski et al. 1996, Niedbała 2008), GR, SM, Imp5, Imp6.

\section{Steganacarus (Tropacarus) carinatus (C. L. Koch, 1841)}

Distribution: Palaearctis and India.

Localities in NP Wigry: Stary Folwark, Krzywe and Poddubówek, (Olszanowski et al. 1996, Niedbała 2008), BE, BB, GR, SM, Imp1, Imp4a, Imp4b, Imp4c, Imp5, Imp6.

Euphthiracaridae Jacot, 1930

34. Acrotritia ardua (C. L. Koch, 1841)

Distribution: cosmopolitan.

Localities in NP Wigry: Poddubówek, (Niedbała 2008), BE, BB

35. Euphthiracarus cribrarius (Berlese, 1904)

Distribution: Holarctis and Nord of Oriental Region.

Localities in NP Wigry: Poddubówek, (Niedbała 2008), BE, BB, Imp4a, Imp4b, Imp4c.

36. Euphthiracarus monodactylus (Willmann, 1919)

Distribution: Holarctis and New Guinea.

Locality in NP Wigry: Imp4a.

\section{Microtritia minima (Berlese, 1904)}

Distribution: semicosmopolitan (Palaearctis, East of Nearctis, Oriental Region, Hawaii).

Localities in NP Wigry: BB, SM, Imp4c.

Malaconothridae Berlese, 1916

38. Malaconothrus monodactylus (Michael, 1888)

Distribution: Holarctis and Neotropical Region.

Localities in Wigry NP: OL, BE, BB, SM. 
Nothridae Berlese, 1896

39. Nothrus palustris C. L. Koch, 1839

Distribution: Holarctis, Himalayas and St. Helena Island.

Localities in NP Wigry: GR, Imp1, Imp4a, Imp4b, Imp4c, Imp6.

40. Nothrus pratensis Sellnick, 1928

Distribution: Holarctis.

Localities in Wigry NP: BE, BB, GR, SM

\section{Nothrus silvestris Nicolet, 1855}

Distribution: Holarctis, Nepal, Mexico and New Zealand.

Localities in NP Wigry: BE, BB, Imp1, Imp4a, Imp4b, Imp5, Imp6.

Camisiidae Oudemans, 1900

42. Heminothrus targionii (Berlese, 1885)

Distribution: Holarctis, China, Nepal and Panama.

Localities in NP Wigry: GR, Imp4a, Imp4b, Imp4c, Imp5, Imp6.

\section{Heminothrus thori (Berlese, 1904)}

Distribution: Holarctis (Palearctis, Nearctis, South-East China, India, Sikkim).

Localities in NP Wigry: OL, BB.

\section{Platynothrus peltifer (C. L. Koch, 1839)}

Distribution: semicosmopolitan (Holarctis, Nord of Oriental Region, New Zealand, Nord of Neotropical Region, St. Helena Island).

Localities in NP Wigry: OL, BE, BB, GR, Imp1, Imp4a, Imp4c, Imp5, Imp6.

Nanhermanniidae Sellnick, 1928

\section{Nanhermannia comitalis Berlese, 1916}

Distribution: Holarctis.

Localities in Wigry NP: OL, BB.

46. Nanhermannia coronata Berlese, 1913

Distribution: Holarctis.

Localities in Wigry NP: BE, BB, SM.

\section{Nanhermannia nana (Nicolet, 1855)}

Distribution: semicosmopolitan (Holarctis, Neotropis, Nord of Oriental Region, New Zealand, St. Helena Island, Antarctica).

Localities in NP Wigry: SM, Imp1, Imp4a, Imp4c.

Hermanniidae Sellnick, 1928

48. Hermannia gibba (C. L. Koch, 1839)

Distribution: Holarctis and Seychelles.

Locality in NP Wigry: Imp4c. 
Hermanniellidae Grandjean, 1934

49. Hermanniella granulata (Nicolet, 1855)

Distribution: Holarctis, South-East China and Nepal.

Localities in NP Wigry: GR, Imp4a, Imp4b, Imp4c, Imp5, Imp6.

Neoliodidae Sellnick, 1928

50. Poroliodes farinosus (C. L. Koch, 1840)

Distribution: Palaearctis and Nepal.

Localities in NP Wigry: SM, Imp4c.

Damaeidae Berlese, 1896

51. Damaeus (Damaeus) auritus C. L. Koch, 1835

Distribution: Palaearctis.

Locality in NP Wigry: Imp4c.

52. Damaeus (Adamaeus) onustus C. L. Koch, 1844

Distribution: West of Palaearctis, Tanzania, St. Helena Island.

Localities in NP Wigry: GR, Imp1,Imp4a, Imp4b, Imp4c, Imp5, Imp6.

53. Damaeobelba minutissima (Sellnick, 1920)

Distribution: Palaearctis.

Localities in NP Wigry: Imp4a, Imp4b.

\section{Metabelba papillipes (Nicolet, 1855)}

Distribution: Holarctis.

Localities in NP Wigry: GR, SM, Imp1, Imp4a, Imp4b, Imp4c, Imp5, Imp6.

\section{Metabelba pulverosa Strenzke, 1953}

Distribution: Holarctis.

Localities in NP Wigry: GR, SM, Imp1, Imp4a, Imp4b, Imp4c, Imp5, Imp6.

56. Metabelba rohdendorfi Bulanova-Zachvatkina, 1965

Distribution: Central and Eastern Europe.

Localities in NP Wigry: SM, Imp1, Imp5.

57. Porobelba spinosa (Sellnick, 1920)

Distribution: Palaearctis.

Localities in NP Wigry: Imp4b, Imp5, Imp6.

Cepheidae Berlese, 1896

58. Cepheus cepheiformis (Nicolet, 1855)

Distribution: Holarctis, South-Eastern China.

Localities in NP Wigry: SM, Imp4a. 
Damaeolidae Grandjean, 1965

59. Fosseremus laciniatus (Berlese, 1905)

Distribution: cosmopolitan.

Localities in NP Wigry: Imp4a, Imp4b, Imp4c, Imp6.

Eremaeidae Oudemans, 1900

60. Eremaeus hepaticus C. L. Koch, 1835

Distribution: Holarctis.

Localities in NP Wigry: GR, Imp1, Imp4a, Imp4b, Imp4c, Imp5, Imp6.

Zetorchestidae Michael, 1898

61. Zetorchestes flabrarius Grandjean, 1951

Distribution: Central and South Europe.

Localities in NP Wigry: GR, Imp4b, Imp4c.

New record for Poland.

Tenuialidae Jacot, 1929

62. Hafenrefferia gilvipes (C. L. Koch, 1839)

Distribution: Palaearctis.

Locality in NP Wigry: Imp4c.

Astegistidae Balogh, 1961

63. Cultroribula bicultrata (Berlese, 1905)

Distribution: Holarctis and Java.

Localities in NP Wigry: BB, SM, Imp1, Imp4a, Imp5.

Liacaridae Sellnick, 1928

64. Adoristes ovatus (C. L. Koch, 1839)

Distribution: Palaearctis.

Localities in NP Wigry: BE, BB, GR, SM, Imp1, Imp4a, Imp4b, Imp4c, Imp5, Imp6.

65. Liacarus coracinus (C. L. Koch, 1841)

Distribution: Palaearctis and St. Helena.

Localities in NP Wigry: Imp4a, Imp4b, Imp4c.

66. Liacarus xylariae (Schrank, 1803)

Distribution: Palaearctic and Nepal.

Locality in NP Wigry: Imp1.

67. Xenillus tegeocranus (Hermann, 1804)

Distribution: Palaearctis and South-Eastern China.

Localities in NP Wigry: GR, Imp1, Imp4a, Imp4c, Imp5, Imp6. 
Carabodidae C. L. Koch, 1837

68. Carabodes coriaceus C. L. Koch, 1835

Distribution: Western Palaearctis and USA.

Localities in NP Wigry: Imp4a, Imp4b, Imp4c, Imp6.

69. Carabodes femoralis (Nicolet, 1855)

Distribution: Eastern, Western and Southern Palaearctis.

Locality in NP Wigry: Imp4a.

70. Carabodes labyrinthicus (Michael, 1879)

Distribution: Holarctis and Mexico.

Locality in NP Wigry: Imp6.

71. Carabodes ornatus Štorkán, 1925

Distribution: Palaearctis.

Localities in NP Wigry: BB, Imp4a, Imp4b, Imp4c.

72. Carabodes rugosior Berlese, 1916

Distribution: Holarctis.

Locality in NP Wigry: Imp4c.

73. Carabodes subarcticus Trägårdh, 1902

Distribution: Palaearctis.

Locality in Wigry NP: SM.

74. Carabodes tenuis Forsslund, 1953

Distribution: Palaearctis and Pakistan.

Locality in Wigry NP: BB.

Tectocepheidae Grandjean, 1954

75. Tectocepheus minor Berlese, 1903

Distribution: semicosmopolitan.

Locality in NP Wigry: Imp5.

76. Tectocepheus velatus (Michael, 1880)

Distribution: cosmopolitan.

Localities in NP Wigry: Poddubówek, (Olszanowski et al. 1996), OL, BB, GR, SM, Imp1, Imp4a, Imp4b, Imp4c, Imp5, Imp6.

Quadroppiidae Balogh, 1983

77. Quadroppia monstruosa Hammer, 1979

Distribution: Holarctis and Java.

Localities in NP Wigry: Imp1, Imp4a, Imp4b, Imp4c, Imp5, Imp6.

New record for Poland.

\section{Quadroppia quadricarinata (Michael, 1885)}

Distribution: semicosmopolitan (Holarctis, South-Eastern China, Mauritius).

Localities in NP Wigry: BE, GR, Imp1, Imp4a, Imp4b, Imp4c, Imp5, Imp6. 
Oppiidae Sellnick, 1937

79. Berniniella bicarinata (Paoli, 1908)

Distribution: Palaearctis, Madagascar and Vietnam.

Localities in NP Wigry: Imp4b, Imp4c.

80. Berniniella sigma (Strenzke, 1951)

Distribution: West Palaearctis, Central and Western Asia.

Locality in NP Wigry: Imp4b.

\section{Dissorhina ornata (Oudemans, 1900)}

Distribution: Holarctis and Africa.

Localities in NP Wigry: Imp4b, Imp5.

\section{Microppia minus (Paoli, 1908)}

Distribution: cosmopolitan.

Localities in NP Wigry: OL, GR, SM, Imp1, Imp4a, Imp4b, Imp4c, Imp5, Imp6.

83. Multioppia glabra (Mihelčič, 1955)

Distribution: Central and Eastern Europe and Eastern Siberia.

Localities in NP Wigry: Imp1, Imp4a, Imp4b, Imp5, Imp6.

\section{Oppiella (Oppiella) falcata (Paoli, 1908)}

Distribution: Palaearctis.

Localities in NP Wigry: OL, GR, SM, Imp1, Imp4a, Imp4b, Imp4c, Imp5, Imp6.

85. Oppiella (Oppiella) nova (Oudemans, 1902)

Distribution: cosmopolitan.

Localities in NP Wigry: OL, BE, BB, GR, SM, Imp1, Imp4a, Imp4b, Imp4c, Imp5, Imp6.

86. Oppiella (Moritzoppiella) neerlandica (Oudemans, 1900)

Distribution: Holarctis.

Localities in NP Wigry: Imp4a, Imp5, Imp6.

87. Oppiella (Moritzoppia) unicarinata (Paoli, 1908)

Distribution: Holarctis and Northern Neotropis.

Locality in NP Wigry: Imp4c.

88. Oppiella (Rhinoppia) subpectinata (Oudemans, 1900)

Distribution: Holarctis and Senegal.

Localities in NP Wigry: BE, BB, SM, Imp4a, Imp4b, Imp4c, Imp5, Imp6.

Suctobelbidae Jacot, 1938

89. Suctobelba regia Moritz, 1970

Distribution: Europe.

Localities in NP Wigry: Imp4a, Imp4b, Imp4c, Imp5, Imp6.

New record for Poland. 
Distribution: Holarctis.

Localities in NP Wigry: BE, BB, Imp1, Imp4a, Imp4b, Imp4c, Imp5, Imp6.

\section{Suctobelbella falcata (Forsslund, 1941)}

Distribution: semicosmopolitan (Holarctis, Salvador, Vietnam and New Zealand).

Localities in NP Wigry: BE, BB, SM, Imp4a, Imp4b, Imp4c, Imp5, Imp6.

92. Suctobelbella forsslundi (Strenzke, 1950)

Distribution: Palaearctis.

Localities in NP Wigry: OL, Imp1, Imp4a, Imp4c, Imp5, Imp6.

\section{Suctobelbella longirostris (Forsslund, 1941)}

Distribution: Holarctis.

Locality in NP Wigry: Imp6.

\section{Suctobelbella nasalis (Forrslund, 1941)}

Distribution: Palaearctis and New Zealand.

Locality in NP Wigry: Imp4a.

95. Suctobelbella palustris (Forsslund, 1953)

Distribution: Holarctis.

Localities in Wigry NP: GR, Imp1.

96. Suctobelbella sarekensis (Forsslund, 1941)

Distribution: Holarctis.

Localities in NP Wigry: BE, BB, Imp1, Imp4a, Imp4b, Imp4c, Imp5, Imp6.

\section{Suctobelbella similis (Forsslund, 1941)}

Distribution: Palaearctis.

Localities in NP Wigry: SM, Imp4b, Imp5, Imp6.

\section{Suctobelbella subcornigera (Forsslund, 1941)}

Distribution: semicosmopolitan (Holarctis, Oriental Region and New Zealand).

Localities in NP Wigry: OL, BE, BB, GR, SM, Imp1, Imp4a, Imp4b, Imp4c, Imp5, Imp6.

99. Suctobelbella subtrigona (Oudemans, 1916)

Distribution: Holarctis, Mexico and Vietnam.

Localities in NP Wigry: BB, Imp4c, Imp5, Imp6.

Autognetidae Grandjean, 1960

100. Autogneta longilamellata (Michael, 1885)

Distribution: Holarctis.

Locality in NP Wigry: Imp1.

101. Conchogneta dalecarlica (Forsslund, 1947)

Distribution: Palaearctis.

Localities in NP Wigry: SM, Imp4a, Imp4c,Imp5, Imp6. 
102. Conchogneta traegardhi (Forsslund, 1947)

Distribution: Holarctis.

Locality in Wigry NP: BB.

Thyrisomidae Grandjean, 1954

103. Banksinoma lanceolata (Michael, 1885)

Distribution: Palaearctis, Canada, and Hawaii.

Localities in NP Wigry: OL, Imp4c.

104. Pantelozetes paolii (Oudemans, 1913)

Distribution: Holarctis and Java.

Locality in NP Wigry: Imp4a.

Phenopelopidae Petrunkevitch, 1955

105. Eupelops acromios (Hermann, 1804)

Distribution: semicosmopolitan.

Localities in NP Wigry: Imp4b, Imp4c.

106. Eupelops hirtus (Berlese, 1916)

Distribution: Holarctis.

Localities in NP Wigry: BB, SM, Imp4c, Imp6.

107. Eupelops plicatus (C. L. Koch, 1835)

Distribution: Holarctis.

Locality in NP Wigry: Imp4c.

108. Eupelops torulosus (C. L. Koch, 1839)

Distribution: Palaearctis and Ethiopia.

Localities in NP Wigry: BE, SM, Imp4b.

Achipteriidae Thor, 1929

109. Achipteria coleoptrata (Linnaeus, 1758)

Distribution: Holarctis, Sikkim, St. Helena Island.

Localities in NP Wigry: BE, GR, Imp1, Imp4a, Imp4b, Imp4c, Imp5, Imp6.

110. Parachipteria punctata (Nicolet, 1855)

Distribution: Holarctis and St. Helena Island.

Locality in NP Wigry: Imp6.

Oribatellidae Jacot, 1925

111. Oribatella calcarata (C. L. Koch, 1835)

Distribution: Holarctis.

Locality in NP Wigry: Imp4c. 
Distribution: Holarctis

Locality in NP Wigry: Imp4b.

Galumnidae Jacot, 1925

113. Acrogalumna longipluma (Berlese, 1904)

Distribution: semicosmopolitan.

Locality in Wigry NP: BE.

114. Galumna lanceata (Oudemans, 1900)

Distribution: Palaearctis and Vietnam.

Localities in NP Wigry: GR, Imp1, Imp4a, Imp5.

\section{Galumna obvia (Berlese, 1915)}

Distribution: semicosmopolitan (Palaearctis, USA, South Africa, Isl. St. Helena, Vietnam, Hawaii and Neotropical Region).

Localities in NP Wigry: OL, BB, Imp4a.

116. Pergalumna nervosa (Berlese, 1914)

Distribution: Holarctis and South Africa.

Locality in NP Wigry: Imp4b.

Ceratozetidae Jacot, 1925

\section{Ceratozetes gracilis (Michael, 1884)}

Distribution: cosmopolitan (Holarctis, St. Helena Island, Oriental, New Zealand, Neotropis, South Shetland Islands).

Localities in NP Wigry: Imp4a, Imp4b, Imp4c, Imp5.

\section{Ceratozetes mediocris Berlese, 1908}

Distribution: semicosmopolitan.

Locality in NP Wigry: Imp4a.

119. Ceratozetes minutissimus Willmann, 1951

Distribution: Central and Southern Palaearctis.

Locality in NP Wigry: Imp4b.

\section{Diapterobates humeralis (Hermann, 1804)}

Distribution: Holarctis.

Locality in NP Wigry: Imp4b.

121. Fuscozetes setosus (C. L. Koch, 1839)

Distribution: Holarctis.

Localities in Wigry NP: BE, BB, SM, Imp4c.

122. Melanozetes mollicomus (C. L. Koch, 1839)

Distribution: Holarctis.

Locality in NP Wigry: Imp4a. 
Chamobatidae Grandjean, 1954

123. Chamobates borealis (Trägårdh, 1902)

Distribution: Palaearctis.

Locality in NP Wigry: Imp1.

124. Chamobates cuspidatus (Michael, 1884)

Distribution: Holarctis and Seychelles.

Localities in NP Wigry: BB, GR, SM, Imp1, Imp4a, Imp4b, Imp4c, Imp5, Imp6.

125. Chamobates subglobulus (Oudemans, 1900)

Distribution: Palaearctis.

Localities in NP Wigry: Imp4b, Imp6.

126. Chamobates voigtsi (Oudemans, 1902)

Distribution: Palaearctis.

Localities in NP Wigry: Imp4c, Imp6.

Mycobatidae Grandjean, 1954

127. Minunthozetes pseudofusiger (Schweizer, 1922)

Distribution: Palaearctis.

Locality in Wigry NP: SM.

128. Minunthozetes semirufus (C. L. Koch, 1841)

Distribution: Holarctis.

Localities in Wigry NP: OL, SM.

129. Punctoribates punctum (C. L. Koch, 1839)

Distribution: semicosmopolitan (Palaearctis, USA, Oriental and New Zealand).

Localities in NP Wigry: Imp4a, Imp4b, Imp5, Imp6.

Euzetidae Grandjean, 1954

130. Euzetes globulus (Nicolet, 1855)

Distribution: Palaearctis and Argentina.

Localities in NP Wigry: BE, BB, GR, SM, Imp1, Imp4a, Imp4b, Imp4c, Imp5.

Haplozetidae Grandjean, 1936

131. Lagenobates lagenulus (Berlese, 1904)

Distribution: Holarctis.

Locality in Wigry NP: BB, SM.

Parakalummidae Grandjean, 1936

132. Neoribates aurantiacus (Oudemans, 1914)

Distribution: Holarctic and Oriental Regions.

Localities in NP Wigry: Imp4a, Imp4b, Imp4c. 
Scheloribatidae Grandjean, 1933

\section{Hemileius initialis (Berlese, 1908)}

Distribution: semicosmopolitan (Palaearctis, USA, Bali and Neotropis).

Locality in NP Wigry: Imp4b.

\section{Liebstadia similis (Michael, 1888)}

Distribution: Holarctis, Sikkim and New Zealand.

Locality in NP Wigry: Imp4c.

\section{Scheloribates laevigatus (C. L. Koch, 1835)}

Distrubution: semicosmopolitan (Holarctic, Argentina and Bolivia).

Localities in NP Wigry: BE, BB, SM, Imp4c.

\section{Scheloribates latipes (C. L. Koch, 1844)}

Distribution: semicosmopolitan (Holarctis, Oriental and Ethiopian Regions).

Localities in NP Wigry: BB, BE.

Oribatulidae Thor, 1929

\section{Oribatula tibialis (Nicolet, 1855)}

Distribution: Holarctis and India.

Localities in NP Wigry: Imp4b, Imp4c, Imp6.

\section{Phauloppia lucorum (C. L. Koch, 1841)}

Distribution: Holarctis and Nepal.

Locality in NP Wigry: Imp5.

\section{Zygoribatula exilis (Nicolet, 1855)}

Distribution: Holarctis and St. Helena Island.

Localities in NP Wigry: Imp4b, Imp4c.

140. Zygoribatula frisiae (Oudemans, 1916)

Distribution: Holarctis.

Locality in NP Wigry: Imp4c.

\section{CONCLUSIONS}

Altogether 280 quantitative soil samples were proceseed during 2016-2019 in 11 localities on the territory of Wigry National Park. Rich material of 25673 individuals of oribatid mites was obtained. In total 140 oribatid species were found in the territory of Wigry NP so far, belonging to 42 families and 80 genera or subgenera. The total number of oribatid mites species found on studied plots was as follows: BB - 36, BE - 26, GR - 34, OL - 15, SM - 38, Imp1 - 44, Imp5 52, Imp $6-50$, Imp4a - 58, Imp4b - 60, Imp4c - 72. Records of six species: Acrotritia ardua, Euphthiracarus cribrarius, Sellnickochthonius jacoti, Steganacarus applicatus, S. carinatus, and Tectocepheus velatus, known earlier from NP Wigry were confirmed, other 127 oribatid species were found on the territory od NP Wigry for the first time and finaly the records of four oribatid mites: Brachychthonius impressus, Quadroppia monstruosa, Suctobelba regia and Zetorchestes flabrarius are new for the fauna of Poland. 


\section{ACKNOWLEDGEMENTS}

The author is grateful for help and field assistance to Drs Anna and Lech Krzystofiak (Krzywe, Poland), Dr Karel Tajovský and Prof. Václav Pižl (both České Budějovice, Czech Republic). The studies on oribatid mites were carried out within the framework of the project: "The influence of Impatiens parviflora on the species diversity of selected organisms and the forest environment of the Wigry National Park", co-financed from the forest fund under the agreement concluded between the State Forests National Forest Holding and the Wigry National Park (EZ.0290.1.20.2019 - Action No 41) and supported also by Czech Academy of Sciences (Research Plan No. RVO 600773644).

\section{REFERENCES}

ABBOtT D. T. \& CROSSLEY D.A. JR. 1982. Woody litter decomposition following clear-cutting. Ecology 63: 35-42. DOI: $10.2307 / 1937028$

Balogh J. \& MahunKa S. 1983. Primitive oribatids of the Palaerctic Region. Akademia Kiadó, Budapest, 372 pp.

Beare M. H., Parmelee R. W., Hendrix P. F., Cheng W., Coleman D. C. \& Crossley D. A. JR. 1992. Microbial and faunal interractions and effects on litter nitrogen and decomposition in agroecosystems. Ecological Monographs 62 : 569-591. DOI: https://doi.org/10.2307/2937317

Behan-Pelletier V. M. \& NeWTON G. 1999. Linking soil biodiversity and ecosystem function - taxonomic dilemma Bioscience 49: 149-153. DOI: https://doi.org/10.2307/1313540

GiLjarov M. S. (ed.) 1975. Opredelitel obitajuščich v počve kleščej. Sarcoptiformes. Nauka, Moscow, 492 pp.

Heneghan L., Coleman D. C., Zhou X., CROSSley D. A. JR. \& HAines B. L. 1999. Soil microarthropod contributions to decomposition dynamics: tropical-temperate comparisons of a single substrate. Ecology 80: 1873-1882. DOI: $10.2307 / 176665$

Kunst M. 1971. Nadkohorta pancíŕníci - Oribatei. In: DANIEL M. \& ČERNÝ V. [eds.], Klíč zvířeny ČSSR, Vol. 4. Academia, Prague, pp. 531-580.

Loreau M., Naeem S., Inchausti P., Bengtsson J., Grime J. P., Hector A., Hooper D. U., Huston M. A., RafFAelli D., Schmid B., Tilman D. \& WARdLE D. A. 2001. Biodiversity and ecosystem functioning: current knowledge and future challenges. Science 294: 804-808. DOI: 10.1126/science.1064088

ŁOZIŃSKI J. 2002. Zmiany drzewostanów objętych ochroną ścisłą w Wigierskim Parku Narodowym. Prace Instytutu Badawczego Leśnictwa, Seria A, 1 (929): 53-74.

MARAUN M., VISSER S. \& SCHEU S. 1998. Oribatid mites enhance the recovery of the microbial community after a strong disturbance. Applied Soil Ecology 9: 175-181. DOI: 10.1016/S0929-1393(98)00072-9

MARShALL V.G. 1972. Comparison of two methods of estimating efficiency of funnel extractors for soil microarthropods. Soil Biology and Biochemistry 4: 417-426. DOI: 10.1016/0038-0717(72)90056-9

NiedBaŁA W. 1976. Brachychthoniidae Polski (Acari, Oribatei). Studium Ekologiczno-faunisticzne. Panstwowe Wydawnictwo Naukowe, Kraków, 145 pp.

NiedbatA W. 2008. Ptyctimous mites (Acari: Oribatida) of Poland. Fauna Polski. Vol. 3, Warszawa, 1-242.

NiedBatA W. 2011. Ptyctimous mites (Acari: Oribatida) of the Palaearctic Region. Systematic part. Fauna Mundi. Vol. 4, Natura Optima, Dux, Warszawa, 472 pp.

Niedbala W. \& OlsZanowski Z. 2008. Mechowce (Oribatida), pp 79-93. In: Bogdanowicz W., ChudZICKA E., PILIPIUK I. \& SKIBIŃSKA E. (eds), Fauna of Poland. Characteristics and checklist of species. Vol. 3. Muzeum Instytut Zoologii PAN, Warszawa, 603 pp.

NORTON R. A. 1985. Aspects of the biology and systematics of soil arachnids, particularly saprophagous and mycophagous mites. Quaestiones Entomologicae 21: 523-541.

OlsZANOWSKi Z., RAJSKi A. \& NiedBAŁA W. 1996. Roztocze (Acari). Mechowce (Oribatida). Katalog fauny Polski 34 (9): 1-243. Sorus, Poznań.

Paoletti M. G., Osler G. H. R., Kinnear A., Black D. G., Thomson L. J., Tsitsilas D., Sharley D., Judd S., NEVILLE P. \& INCA A. D. 2007. Detritivores as indicators of landscape stress and soil degradation. Australian Journal of Experimental Agriculture 47: 412-423.

PETERSEN H. \& LUXTON M. 1982. A comparative analysis of soil fauna populations and role in decomposition processes. Oikos 39: 287-388.

SCHATZ H. 2002. Die Oribatidenliteratur und die beschreibenen Oribatidenarten (1758-2001) Eine Analyse. Abhandlungen und Berichte des Naturkundemuseum Gorlitz 74: 37-45.

SEASTEDT T. R. 1984. Microarthropods of burned and unburned tallgrass prairie. Journal of the Kansas Entomological Society 57: 468-476.

SEASTEDT, T. R. \& CROSSLEY D. A. JR. 1988. Soil arthropods and their role in decomposition and mineralization processes. In: SwANK W. T. \& CROSSLEY D. A. JR. (eds), Ecological Studies, 66. Forest Hydrology and Ecology at Coweeta. Springer, Athens, Georghia, USA, pp. 233-244. 
SELlNICK M. 1928. Formenkreis: Hornmilbenart, Oribatei. In: BROHMER J., EHRMAN K. \& UlmER C. (eds), Die Tierwelt Mitteleuropas, Vol. 3 (9). 42 pp.

SEllnick M. 1960. Oribatei. In: Brohmer J., Ehrman K. \& Ulmer C. (eds), Die Tierwelt Mitteleuropas, Vol. 3 (4). pp. $45-136$.

SETÄLÄ H. \& HuHTA V. 1991. Soil fauna increase Betula pendula growth - laboratory experiments with coniferous forest floor. Ecology 72: 665-671. DOI: https://doi.org/10.2307/2937206

SUBIAS L.S. 2004. Listado sistemático, sinonímico y biogeográfico de los ácaros oribátidos (Acariformes, Oribatida) del mundo (1758-2002). Graellsia 60: 3-305. DOI: 10.3989/graellsia.2004.v60.iExtra.218

WeigmanN G. 2006. Hornmilben (Oribatida). Acari, Actinotrichida. In: Die Tierwelt Deutschlands, Vol. 76. Goecke \& Evers, Keltern, 520 pp.

WiLLMANN K. 1931. Moosmilben oder Oribatiden (Oribatei). In: Die Tierwelt Deutschlands, Vol. 22. pp. 79-200.

\section{STRESZCZENIE}

[Lista gatunków mechowców (Acari: Oribatida) Wigierskiego Parku Narodowego, Polska północno-wschodnia]

Szczegółowe badania faunistyczne nad glebowymi mechowcami (Acari: Oribatida) przeprowadzono na 11 stanowiskach reprezentujących istotne typy środowisk leśnych w obszarze Wigierskiego Parku Narodowego. Łącznie wykazano 140 gatunków mechowców należących do 42 rodzin i 80 rodzajów lub podrodzajów. Spośród nich 127 gatunków stwierdzono na obszarze Wigierskiego Parku Narodowego po raz pierwszy, w tym cztery gatunki, tj. Brachychthonius impressus Moritz, 1976, Quadroppia monstruosa Hammer, 1979, Suctobelba regia Moritz, 1970 and Zetorchestes flabrarius Grandjean, 1951 okazały się być nowe w faunie Polski.

Accepted: 20 March 2020 